\title{
A framework to design and solve Markov Decision Well-formed Net models
}

\author{
M. Beccuti, D. Codetta-Raiteri, G. Franceschinis * \\ Dip. di Informatica, Univ. del Piemonte Orientale \\ Alessandria, Italy \\ $\{$ beccuti, raiteri, giuliana $\} @$ mfn.unipmn.it
}

\author{
Serge Haddad ${ }^{\dagger}$ \\ LAMSADE CNRS, Univ. de Paris Dauphine \\ Paris, France \\ haddad@lamsade.dauphine.fr
}

\section{Introduction}

The Markov Decision Process (MDP) [7] formalism is widely used for modeling systems which exhibit both non deterministic and probabilistic behaviors (e.g. distributed systems, resource management systems, ...). Unfortunately, if the system is particularly complex then its modeling at the MDP level may be very hard; so in [6] a higherlevel formalism called Markov Decision Well-formed Net (MDWN) was proposed. The MDWN allows to describe the system in terms of its components and their interactions, while the MDP describes directly the state space and the state transitions. The MDWN model is more compact and readable: in particular, it is possible to define a complex non deterministic or probabilistic behavior as a composition of simpler non deterministic or probabilistic steps. In the MDWN formalism, the probabilistic behavior of the system is clearly distinct from the non deterministic one; actually they are designed as two separate Petri Nets (PN): the probabilistic PN $\left(N^{p r}\right)$ and the non deterministic PN $\left(N^{n d}\right)$.

From the analysis point of view, the MDWN allows to derive efficient algorithms (e.g. taking advantage from the intrinsic symmetries of the system) reducing the analysis complexity. In fact the technique of Symbolic Reachability Graph (SRG) developed for the Well-formed Nets (WNs) [1] was adapted for the MDWN so that a reduced MDP (whose states represent aggregates of equivalent ordinary states) can be produced and directly used to compute the solution of the original problem. In this paper, we introduce the MDWNsolver framework for the system modeling and the optimization of performability measures based on the MDWN formalism. This framework combines together several tools: Draw-Net [3] for the model design, algebra to compose the non deterministic PN with the probabilistic one, $W N(S) R G$ to build the (S)RG of the resulting com-

${ }^{*}$ The work of these authors was partially supported by Consorzio Nazionale Interuniversitario per le Telecomunicazioni (CNIT), under the EU CRUTIAL project.

$\dagger$ The work of this author was supported in part by ANR-06-SETI-002 project Checkbound. posed net, $R G 2 M D P$ to generate the corresponding MDP from the (S)RG, and MDPsolver based on the library graph $M D P[5]$ to compute the optimal strategy and the optimal average reward. The tools algebra and $W N(S) R G$ belong to the GreatSPN suite [2]. In the following section we are going to describe this architecture with more details.

\section{Framework architecture}

The architecture of our framework for the MDWN design and solution, is depicted in Fig. 1 where the framework components are indicated by rectangles, the component invocations are shown as thick arrows, while the exchange of models and of data is represented by thin arrows.

The Draw-Net Modeling System (DMS) [3] is exploited as graphical interface and as solution manager. The $D M S$ model editor called Draw-Net [3] allows the user to design the $N^{p r}$ and the $N^{n d}$ and to define the reward function. To this aim, the Draw-Net tool has been adapted to draw such models by defining an ad-hoc formalism [3]. The $D M S$ is able to save each PN in the GreatSPN file format (.net and def file) [2], by means of an ad-hoc filter. The solution manager of the $D M S$ is exploited to execute in the correct order the framework components, and to manage the exchange of models and of data between them. The solution process comprises four steps:

(1) The $N^{p r}$ and the $N^{n d}$ models are composed by using the algebra tool: this requires to automatically generate a Composition Net and to perform a composition by place superposition [6]. The result of this step is a WN.

(2) The WN is the input of $W N(S) R G$ generating the (S)RG. After this, each probabilistic path in the $(\mathrm{S}) \mathrm{RG}$ is substituted by a single transition. The resulting graph is stored in a file (.mdwn).

(3) From the graph obtained in step 2 an MDP is derived by means of the $R G 2 M D P$ converter using the reward definition. In case multi-step non deterministic decisions are present in the model, these are reduced to a single step complex decision.

(4) The obtained MDP is stored in an XML file which 


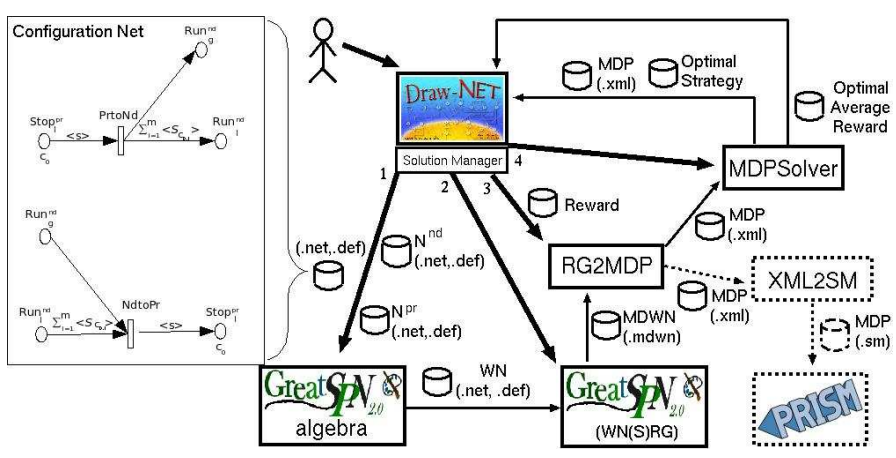

Figure 1. Framework architecture.

is in turn processed by the MDPSolver producing the $o p$ timal strategy and the optimal average reward. The MDP together with the optimal strategy, and the optimal average reward can be visualized by Draw-Net.

We are also planning the integration of PRISM [4] in our framework, with the role of alternative solver or model checker of the MDP. This is represented by the dashed part in the architecture shown in Fig. 1.

\section{Application example}

An implementation of such framework is available and some experimental results are now available: for instance in [6] a model representing a multiprocessor system was presented. The system is composed by several couples of processor and local memory with the addition of one global memory which may fail and can be repaired. A processor can use the global memory when its local memory is faulty. The probabilistic part represents the component faults while the non deterministic part represents the component repair strategy. A cost is associated with every component repair and a penalty is defined when the system is not available; the goal is to find an optimal strategy minimizing the system costs (repair + penalty).

The memory and time requirements for the solution of this MDWN are summarized in Table 1. The table shows

\begin{tabular}{|c||r|r||r|r||c|c||}
\hline \multicolumn{1}{|c||}{} & \multicolumn{2}{c||}{ Proc,Mem=2 } & \multicolumn{2}{c||}{ Proc,Mem=3 } & \multicolumn{2}{c||}{ Proc,Mem=4 } \\
\hline & \#States & \multicolumn{1}{c|}{ Time } & \#States & Time & \#States & Time \\
\hline \hline RG & 40.088 & $6 \mathrm{~s}$ & 1.619 .392 & $1.367 \mathrm{~s}$ & 58.741 .760 & $\begin{array}{c}\text { out of } \\
\text { memory }\end{array}$ \\
\hline MDP & 441 & $7 \mathrm{~s}$ & 4.078 & $5.183 \mathrm{~s}$ & $\begin{array}{c}\text { out of } \\
\text { mem }\end{array}$ & $\begin{array}{c}\text { out of } \\
\text { mem }\end{array}$ \\
\hline \hline SRG & 20.316 & $5 \mathrm{~s}$ & 283.128 & $284 \mathrm{~s}$ & 2.734 .826 & $2.477 \mathrm{~s}$ \\
\hline MDP $^{\prime}$ & 219 & $4 \mathrm{~s}$ & 831 & $534 \mathrm{~s}$ & 2.360 & $48.722 \mathrm{~s}$ \\
\hline
\end{tabular}

Table 1. Number of states and computation time of the example described in [6].

the number of states and the computation time of the RG, the corresponding MDP, the SRG and the corresponding $\mathrm{MDP}^{\prime}$ for different numbers of processors and memories, performed with an AMD Athlon $642.4 \mathrm{Ghz}$ with $4 \mathrm{~Gb}$ memory capacity. The computation time of MDP/MDP' is the sum of their generation and solution time.

By comparing the RG line with the SRG line it is possible to appreciate the reduction of memory and time requirements due to the state aggregation obtained through the SRG technique. The difference between the (S)RG size and the corresponding MDP size, suggests that the modeler has exploited the possibility to decompose a complex non deterministic/probabilistic state transition into simpler steps (referred to a single component rather than to a system as a whole): as the number of components grows, this may became a crucial aspect w.r.t. the usability of the formalism.

\section{References}

[1] G. Chiola, C. Dutheillet, G. Franceschinis, and S. Haddad. Stochastic well-formed coloured nets for symmetric modelling applications. IEEE Transactions on Computers, 42(11):1343-1360, November 1993.

[2] G. Chiola, G. Franceschinis, R. Gaeta, and M. Ribaudo. GreatSPN 1.7: Graphical editor and analyzer for timed and stochastic petri nets. Performance Evaluation, special issue on Performance Modeling Tools, 24(1-2):4768, November 1995.

[3] D. Codetta-Raiteri, G. Franceschinis, and M. Gribaudo. Defining formalisms and models in the Draw-Net Modelling System. In 4th Int. Workshop on Modelling of Objects, Components and Agents (MOCA), pages 123 144, Turku, Finland, June 2006.

[4] A. Hinton, M. Kwiatkowska, G. Norman, and D. Parker. PRISM: A tool for automatic verification of probabilistic systems. In 12th Int. Conf. on Tools and Algorithms for the Construction and Analysis of Systems, volume 3920 of LNCS, pages 441-444, Vienna, Austria, 2006. Springer.

[5] P. Laroche. Graphmdp: A new decomposition tool for solving markov decision processes. Int. Journal on Artificial Intelligence Tools, 10(3):325-343, 2001.

[6] M. Beccuti, G. Franceschinis, and S. Haddad. Markov Decision Petri Net and Markov Decision Well-Formed Net Formalisms. In 28th Int. Conf. on application and theory of Petri nets and other models of concurrency (ICATPN) 2007, volume 4546 of LNCS, pages 43-62. Springer-Verlag Berlin Heidelberg, 2007.

[7] M. L. Puterman. Markov Decision Processes. Discrete Stochastic Dynamic Programming. Wiley, 2005. 